\title{
Development of a Robust Screening Method for Pathogenicity of Colletotrichum spp. on Strawberry Seedlings Enabling Forward Genetic Studies
}

\author{
Sigal Horowitz, Department of Plant Pathology and Microbiology, Faculty of Agricultural, Food and Environmental \\ Quality Sciences, The Hebrew University of Jerusalem, Rehovot 76100, and Department of Plant Pathology, ARO, \\ The Volcani Center, Bet Dagan 50250, Israel; Oded Yarden, Department of Plant Pathology and Microbiology, Fac- \\ ulty of Agricultural, Food and Environmental Quality Sciences, The Hebrew University of Jerusalem; and Aida \\ Zveibil and Stanley Freeman, Department of Plant Pathology, ARO, The Volcani Center
}

\begin{abstract}
Horowitz, S., Yarden, O., Zveibil, A., and Freeman, S. 2004. Development of a robust screening method for pathogenicity of Colletotrichum spp. on strawberry seedlings enabling forward genetic studies. Plant Dis. 88:845-851.

Generation and screening for nonpathogenic mutants is a popular tool for identifying pathogenicity-related genes. Successful application of this technique for plant fungal pathosystems requires reliable and rapid screening procedures. This study reports on the development of a rapid in vitro bioassay enabling large-scale screening and isolation of nonpathogenic mutants of Colletotrichum gloeosporioides and C. acutatum on strawberry seedlings. Inoculation was carried out on strawberry seedlings at two different developmental stages: 12-week-old (young) and 15-week-old (older) seedlings. A comparison was made between two inoculation techniques, (i) foliar dip and (ii) root soak, at two incubation temperatures $\left(19\right.$ and $\left.25^{\circ} \mathrm{C}\right)$. Mortality of young seedlings was observed 4 days after inoculation with both species, reaching $50 \%$ within 10 days, using both techniques at $25^{\circ} \mathrm{C}$. However, mortality of older seedlings was delayed by 4 days compared with that in the young seedlings when using the root-soak method. Disease development decreased in young and older seedlings at the lower temperature. This method also was reliable in determining pathogenicity of the cucurbit-specific C. magna that did not cause disease symptoms on strawberry by either inoculation method. The proposed method enabled screening of more than 980 restriction enzyme-mediated integration mutants resulting in a selection of five reduced-virulence isolates. Initial characterization of some of these mutants revealed large differences in germination and appressorial formation compared with pathogenic isolates.
\end{abstract}

Additional keyword: anthracnose

Anthracnose has become a major constraint in worldwide strawberry (Fragaria $\times$ ananassa Duchesne) production. The dominant species causing disease symptoms on strawberry are Colletotrichum acutatum J. H. Simmonds, C. fragariae Brooks, and C. gloeosporioides (Penz.) Penz. \& Sacc. in Penz. (teleomorph Glomerella cingulata (Stoneman) Spauld. \& H. Schrenk) $(11,15,21,26)$. Anthracnose disease of strawberry affects roots, crowns, petioles, leaves (preferentially immature ones), runners, buds, flowers, and fruit $(6,9,10)$. The ability of these pathogens to attack different plant parts, and the fact that several pathogen species are involved,

Corresponding author: S. Freeman

E-mail: freeman@volcani.agri.gov.il

Contribution from the Agricultural Research Organization, Institute of Plant Protection, Bet Dagan, Israel, No. 519/03. This research was supported in part by grant no. US-3260-01C from BARD, The United States-Israel Binational Agricultural Research and Development Fund.

Accepted for publication 31 March 2004.

Publication no. D-2004-0609-01R

(C) 2004 The American Phytopathological Society genetic approach for screening for reduced or impaired virulence mutants. In general, the following procedures should be available for this purpose: (i) a random mutagenesis system, (ii) an efficient transformation system, and (iii) a rapid, reliable infection bioassay (29). The restriction enzyme-mediated integration (REMI) technique has been applied successfully to isolate pathogenicity-related genes in several plant-pathogenic fungi $(2,3,19,29,31,33)$ and may serve as an insertional mutagenesis method. An efficient transformation system was developed for C. gloeosporioides (25) and in our laboratory for $C$. acutatum (14) by electroporation of germinating conidia, which provided an easy and efficient way to obtain stable transformants of this fungus. However, in the past, pathogenicity assays of Colletotrichum spp. were designed especially for breeding programs for anthracnose resistance and are not suitable for large-scale screening for reduced-pathogenicity mutants. These techniques involve foliar application of conidia by spraying, pipetting, or injection of seedlings or plants, which ultimately were selected for foliage, stolon, and crown resistance $(7,28)$. Such methods require considerable greenhouse space, are time and labor consuming, and carry a high chance of cross contamination during inoculation; thus, they are not appropriate for a large-scale screening procedure for nonpathogenic mutants. Likewise, strawberry fruit inoculation methods have been developed to study the virulence of (22) and resistance to anthracnose fruit rot (8). Similarly, an inoculation technique of detached petioles was developed for assessing resistance of strawberry lines to $C$. acutatum (18). However, these two latter methods, in which detached organs were used, are inappropriate for assessing pathogenicity, because resistance mechanisms in detached tissues may be reduced.

The main objective of this study was to establish a rapid and reliable bioassay for large-scale screening of putative nonpathogenic Colletotrichum mutants on strawberry. The effects of incubation temperature, seedling age, inoculation technique, and isolate were evaluated to determine the conditions best suited for this purpose. A second objective was to prove the efficacy of the proposed screening method by iso- 
lating reduced-pathogenicity mutants for future studies.

\section{MATERIALS AND METHODS}

Fungal isolates and inoculum preparation. C. acutatum isolate C.a 149 (IMI391664) (10) and C. gloeosporioides isolates C.g 318 (IMI391663) and C.g 342 pathogenic on strawberry (13), C. magna isolate L2.5 (IMI391662) pathogenic and an ultraviolet-induced nonpathogenic isolate (n-path) on cucurbits (12) were used as control isolates in the development of the screening procedure. The fungi were maintained in the dark on modified Mathur's medium (M3S) as previously described (10). To prepare inoculum, conidia were washed from 5- to 6-day-old cultures with distilled water and adjusted to a final desired concentration of $10^{4}, 10^{5}$, or $10^{6}$ per $\mathrm{ml}$ with a hemacytometer. Approximately 980 transgenic isolates of $C$. acutatum and $C$. gloeosporioides were generated (see below) and evaluated for pathogenicity. Mutants were maintained on M3S supplemented with hygromycin B at $60 \mu \mathrm{g} / \mathrm{ml}$ (Calbiochem, San Diego, CA).

Propagation of strawberry seedlings. Seed of strawberry cv. Malach, an anthracnose-susceptible strawberry cultivar, were produced from fruit harvested from December to March from a commercially cultivated field (Tzofit, Central Sharon region, Israel). The seeds were collected after peeling and drying the skins for $72 \mathrm{~h}$ at $\pm 25^{\circ} \mathrm{C}$. The seeds then were surfacedisinfested and soaked overnight in sterile deionized water $\left(\mathrm{dH}_{2} \mathrm{O}\right)$. Seeds were germinated on moist, finely ground peat-vermiculite medium (vol/vol; $1: 1$ ) in containers and overhead irrigated with $\mathrm{KNO}_{3}(2$ $\mathrm{g} / \mathrm{liter}$ ) soluble fertilizer solution. Containers were covered with plastic bags to maintain 90 to $100 \%$ relative humidity and kept at $25 \pm 1{ }^{\circ} \mathrm{C}$ under continuous fluorescent light for 2 weeks. Thereafter, the plastic bags were removed and the germinated strawberry seedlings were transferred to a greenhouse with supplemental lighting (16-h photoperiod) and temperature of 25 $\pm 1^{\circ} \mathrm{C}$. Plants in the greenhouse were overhead irrigated each week with a soluble fertilizer (N-P-K 4:2.5:6 + 6\% microelements; Haifa Chemical Corp., Haifa, Israel). After 12 weeks, most of the seedlings used for inoculation experiments consisted of four to five true leaves.

Inoculation techniques. Two inoculation methods were evaluated for their effect on subsequent disease development. The methods included a root soak and foliar dip of strawberry seedlings in conidial suspensions. For the root-soak method, conidial suspensions from isolates C.a 149, C.g 318, and n-path were used separately by inserting seedling roots into $2-\mathrm{ml} \mathrm{Ep-}$ pendorf tubes containing $1.5 \mathrm{ml}$ of conidial suspensions at different concentrations. Seedlings inoculated by foliar dip were inverted and inserted into a conidial sus- pension of the three control isolates and inoculum was applied to all the seedling parts, above the roots. Seedlings then were inserted into Eppendorf tubes filled with $1.5 \mathrm{ml}$ of sterile $\mathrm{dH}_{2} \mathrm{O}$. Sterile water was used as a control for noninoculated seedlings in both methods. To maintain high relative humidity, inoculated and control seedlings were transferred immediately into boxes covered with plastic bags, and incubated for a period of 20 days in environmental chambers at 19 or $25^{\circ} \mathrm{C}$. In each experiment, 50 seedlings were tested for each control isolate at each temperature. Twenty seedlings treated with sterile $\mathrm{dH}_{2} \mathrm{O}$ served as noninoculated controls. Experiments determining the effect of inoculation method on seedling mortality were repeated twice for each control isolate (50 replicate seedlings were tested for each treatment).

Seedling age and disease response. The effect of seedling age on disease response was evaluated at two developmental stages: (i) young, 12-week-old seedlings and (ii) older, 15-week-old seedlings. Both sets of seedlings were grown for the first 12 weeks as described above. Older seedlings were transplanted at 12 weeks to polyethylene trays containing peat-vermiculite medium (vol/vol; $1: 1$ ) and were grown for an additional 3 weeks in a greenhouse with a 16 -h photoperiod. Conidial suspensions of isolates C.a 149, C.g 318 , and n-path were used separately to inoculate young or older seedlings at 19 and $25 \pm 1{ }^{\circ} \mathrm{C}$ and mortality was recorded over a 3-week period. Experiments examining the effect of seedling age were repeated twice for each control isolate at each incubation temperature (50 replicate seedlings were tested for each treatment).

Fungal transformation. Electroporation of germinating conidia was performed essentially as previously described (14). Briefly, isolates C.a 149, C.g 342, and C.g 318 were cultured on solid M3S medium for 5 days. Conidia were collected in pea juice (25), adjusted to $10^{6} \mathrm{conidia} / \mathrm{ml}$, and incubated at $28^{\circ} \mathrm{C}$ for 4.5 to $5 \mathrm{~h}$ to initiate germination. The germinated conidia were collected, washed with cold electroporation buffer (1 $\mathrm{mM}$ n-2-hydroxyethlpiperazine-N-2-ethanesulfonic acid [HEPES], $50 \mathrm{mM}$ mannitol, $\mathrm{pH}$ 7.5) and concentrated to $10^{8}$ conidia $/ \mathrm{ml}$, and $100-\mu \mathrm{l}$ aliquots were distributed in cold electroporation cuvettes (Bio-Rad, Hercules, CA). The 5.13-kb pGH-1 plasmid (33) was used as a transformation vector. REMI was performed with $5 \mu \mathrm{g}$ of linearized XbaI or HindIII pGH-1 plasmid for each transformation event (performed in separate cuvettes) in the presence of 24 units of the same enzyme used to linearize the plasmid, as previously described (33).

Electroporation was performed with a gene pulser (Bio-Rad) operating at $1.4 \mathrm{kV}$, $800 \Omega$, and $25 \mu \mathrm{F}$. After application of the electric pulse, the conidia were transferred to regeneration (Reg) medium (145.7 $\mathrm{g}$ of mannitol, $4 \mathrm{~g}$ of yeast extract [Difco Laboratories, Detroit], $1 \mathrm{~g}$ of soluble starch, 16 $\mathrm{g}$ of agar [Difco Laboratories], and $50 \mathrm{ml}$ of pea juice per liter). After $10 \mathrm{~h}$, a top overlay $(2 \mathrm{~mm})$ of water agar with hygromycin B $125 \mu \mathrm{g} / \mathrm{ml}$ (Calbiochem) was added. Transgenic colonies appeared 4 to 5 days after transformation. Stability of transformants was assessed by serial transfer of mycelial plugs of representative REMI transformants onto fresh Reg medium containing or devoid of hygromycin B at $60 \mu \mathrm{g} / \mathrm{ml}$ every 2 weeks for a 2-month period.

Pathogenicity assays for isolation of putative nonpathogenic mutants. Hygromycin-transformed putative nonpathogenic mutant isolates were tested for their ability to induce anthracnose symptoms on strawberry seedlings. Primary pathogenicity screens used five anthracnose susceptible 'Malach' seedlings per REMI transformant. Young seedlings with four to five true leaves were inoculated by the foliardip method with $10^{5}$ conidia/ml of each REMI isolate and inserted into Eppendorf tubes. Seedling mortality was assessed after 7 to 10 days. Transformants that did not cause disease on any of the five plants were subjected to an additional pathogenicity screening, using 15 seedlings. REMI transformants exhibiting reduced pathogenicity were screened a third time in the same manner as the second test. All experiments included a positive control that consisted of 15 seedlings inoculated with wild-type isolates of C. gloeosporioides, C.g 318, and C.g 342, and $C$. acutatum, C.a 149. Negative controls consisted of 15 replicate seedlings inoculated with n-path of $C$. magna and a water control. Pathogenicity of the putative mutants was verified further on mature daughter plants. Mature plants were inoculated by foliar dip or sprayed with conidial suspensions of $10^{6}$ conidia $/ \mathrm{ml}$ and were maintained under 90 to $100 \%$ relative humidity by covering with plastic bags at $25 \pm 1{ }^{\circ} \mathrm{C}$ for 2 weeks. Disease severity of daughter plants was assessed after 2 weeks according to the following disease severity rating (DSR) scale: $0=$ plants with no visible lesions; $1=$ one small $(<0.5 \mathrm{~cm})$ lesion on leaves or petioles; $2=$ two small lesions on leaves or petioles; $3=$ one or more large, actively developing lesions on leaves or petioles; $4=$ developing necrotic and wilt symptoms; and $5=$ dead plant with necrotic lesions. Pathogenicity of each potential mutant and wild-type isolates was expressed as the mean DSR of 10 mature strawberry plants per isolate.

Germination and appressorial formation. Germination and appressorial formation by Colletotrichum mutants impaired in pathogenicity and comparative wild-type isolates were observed on GLASSTIC SLIDES (HYCOR; Biomedical Inc., Garden Grove, CA). Briefly, $200 \mu \mathrm{l}$ of a conid- 
ial suspension of $10^{5}$ conidia $/ \mathrm{ml}$ of each isolate was placed into five cells of the glasstic slide and kept on moistened filter paper in a moist chamber. After 12 to $14 \mathrm{~h}$ of incubation, conidial germination and appressorial development were observed in three microscopic fields in each of five cells per slide. Three germination and appressorial formation stages were documented: conidial germination and immediate appressorial formation (appressoria), conidial germination and appressorial formation after more than double the conidia length (germination + appressoria), and conidia germination without appressorial formation (conidia + germination). Frequencies of germination and appressorial formation were determined for 400 conidia from each isolate in each of three trials and compared with that of wild-type frequency by a least significance difference test (LSD; $P=0.05$ ). To confirm isolate phenotype, strawberry seedlings were inoculated with conidial droplets and germination and appressorial formation was monitored microscopically after 12 to $14 \mathrm{~h}$ on leaves.

Statistical analysis. Data were analyzed using the JMP software package (version 3.2.6; SAS Institute, Inc. Cary, NC). Each in vitro seedling inoculation experiment was repeated once and data from each experimental repeat were subjected to analysis of variance (ANOVA) and error variances were evaluated for homogeneity by pairwise $t$ tests. ANOVA was performed separately for each seedling age $\times$ incubation temperature $x$ inoculation method $x$ incubation temperature of each isolate combination. Because there were no significant statistical differences among experiments and the error variances were homogeneous $(P>0.10)$, data from individual experiments were combined. The combined data were separated using the Tukey-Kramer multiple comparison test, $P$ $<0.05$, to detect differences among treatments. Additionally, the time period required to reach $50 \%$ seedling mortality was calculated from the respective regression equation of disease progress for each isolate combination. Significant differences of time to reach $50 \%$ mortality were calculated by contrast $t$ tests $(P<0.05)$, in an ANOVA for the linear regression. Frequency of germination and appressorial formation were pooled for each of three replications per isolate after $t$ tests were conducted to determine homogeneity of variance among the three independent experiments, and no significant statistical difference among experiments was found $(P>0.05)$. Mean comparisons of germination and appressorial formation were calculated with the combined data using LSD, according to the Tukey-Kramer multiple comparison test at $P<0.05$.

\section{RESULTS}

Disease incidence in strawberry seedlings. Disease incidence and symptom development in the inoculated seedlings were monitored over 20-day periods. After this period, lower leaves closest to the water began to senesce and rot in water and n-path $C$. magna control treatments. Therefore, the experiments were terminated at this stage. Seedling mortality was determined as a binary test (dead or live seedling) and not by a disease severity rating (DSR) scale, which was used later on mature plants inoculated with putative nonpathogenic mutants. Local anthracnose lesions on leaves and petioles were monitored but seedling mortality was recorded only when all seedling petioles collapsed, which led to seedling death. Disease progress in the seedlings inoculated with the n-path isolate was similar to water controls and was significantly lower than in seedlings inoculated with the pathogenic wildtype isolates (Fig. 1A).

Effect of seedling age on disease response. The effect of seedling age on disease response was evaluated using 12week-old (young) and 15-week-old (older) seedlings. A rapid disease response, similar for both young and older inoculated seed- lings, was observed within 4 days, by the foliar dip method, with isolates C.g 318 of C. gloeosporioides and C.a 149 of C. acutatum (Fig. 2A). No significant differences were observed in the time required to reach $50 \%$ seedling mortality of young and older seedlings inoculated by the foliar dip method at $25^{\circ} \mathrm{C}$ (Table 1). However, seedling mortality decreased significantly in older seedlings compared with young seedlings inoculated by the root-soak method, with all pathogenic isolates (Fig. 2B). Mortality of young seedlings was observed from 4 days after root inoculation, whereas that of older seedlings was delayed, occurring 8 days after inoculation (Fig. 2B).

Effect of inoculation method on disease response. Foliar-dip and root-soak inoculation methods were evaluated for their effect on subsequent disease development. Young and older seedlings inoculated by the foliar-dip method developed typical anthracnose lesions on foliage and petioles within 2 to 3 days. However, seedlings inoculated by the root-soak method did not develop lesions on foliage but ex-
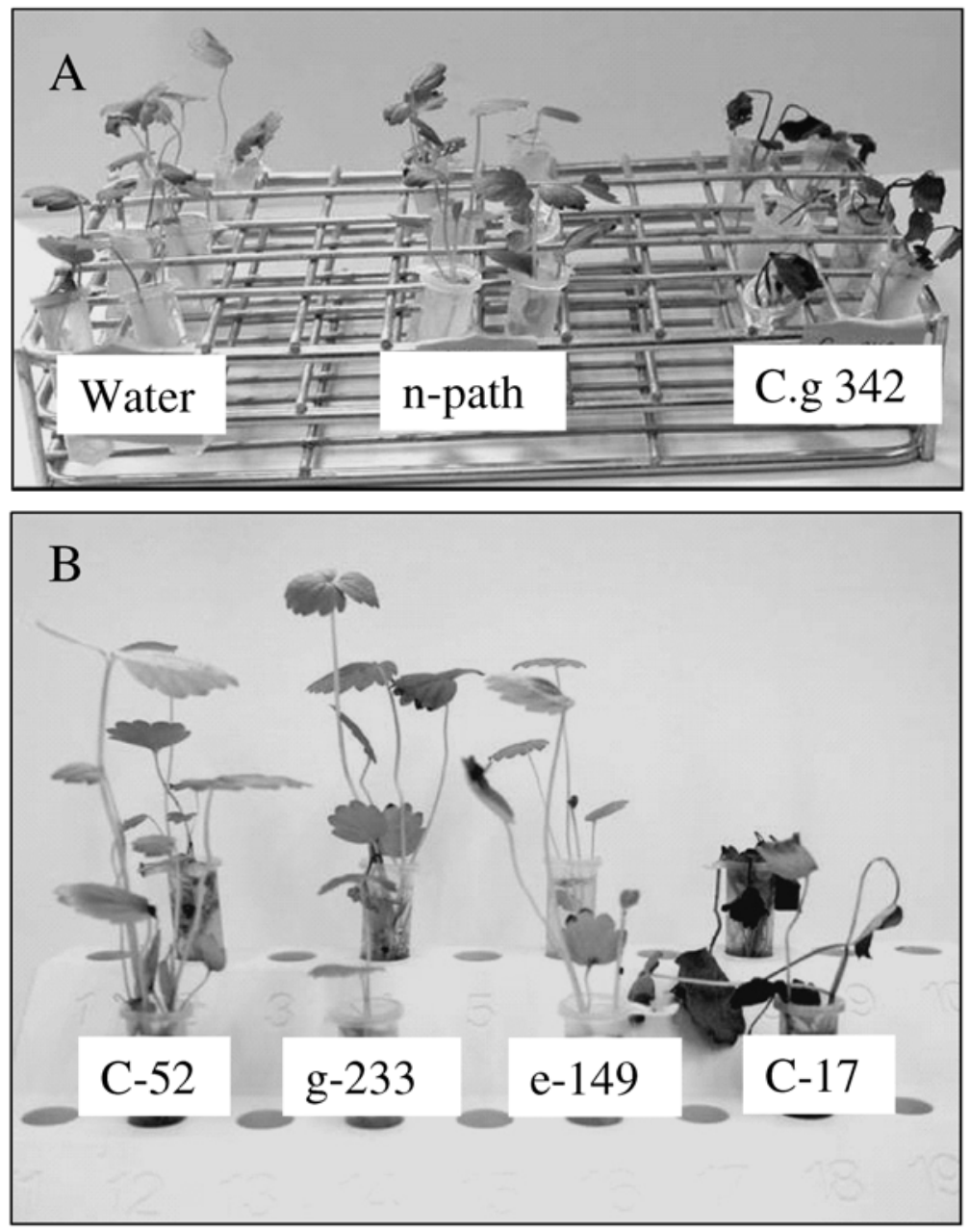

Fig. 1. Disease incidence 16 days after inoculation with A, young seedlings inoculated with a nonpathogenic isolate (n-path) of Colletotrichum magna, pathogenic isolate C.g 342 of C. gloeosporioides, and water control, and B, reduced pathogenicity mutants (c-52, g-233, and e-149) and pathogenic mutant (C-17) of C.g 342 of C. gloeosporioides. Seedlings were inoculated by the foliardip method in a conidial suspension $\left(10^{5}\right.$ conidia/ml $)$ of each isolate and incubated at $25^{\circ} \mathrm{C}$. 
hibited active necrotic lesions on lower petioles that resulted in rapid seedling collapse at $25^{\circ} \mathrm{C}$. The root-soak technique resulted in rapid seedling collapse but minimal anthracnose symptoms were observed on foliage. No significant differences were observed in time required to reach $50 \%$ disease incidence of young seedlings inoculated by the two methods at $25^{\circ} \mathrm{C}$ (Table 1). In older seedlings, foliar dip produced more rapid disease progress than root soak (Fig. 2A and B) and time required for $50 \%$ mortality was significantly less for seedlings inoculated with most isolates by foliar dip than by root soak at $25^{\circ} \mathrm{C}$ (Table 1 ).

Effect of incubation temperature on disease response. Temperatures of 19 and $25^{\circ} \mathrm{C}$ were evaluated for their effect on disease development and pathogenicity levels. The onset of seedling mortality occurred 2 days later at $19^{\circ} \mathrm{C}$ compared with $25^{\circ} \mathrm{C}$ for both young and older seedlings inoculated by foliar dip (Fig. 2A and C). Significant differences in time required to reach $50 \%$ mortality of seedlings were observed only for young seedlings inoculated with C.a 149 by the foliage method at both incubation temperatures (Table 1). Disease progress was much slower for young and older seedlings inoculated at $19^{\circ} \mathrm{C}$ by the root-soak method with isolate
C.g 318 of C. gloeosporioides than in young seedlings inoculated with isolate C.a 149 of C. acutatum (Fig. 2D).

Effect of inoculum concentration on disease response. Inoculum concentrations of $10^{4}, 10^{5}$, and $10^{6}$ conidia/ml of three isolates C.a 149, C.g 318, and n-path of C. magna were assessed for disease response (Table 2). Significant differences in disease progress were observed for isolate C.g 318 of C. gloeosporioides at all three inoculum concentrations. Significant differences also were observed for C.g 318 in the time required to reach $50 \%$ seedling mortality of $13.8,9.5$, and 6.4 days for $10^{4}$, $10^{5}$, and $10^{6}$ conidia/ml, respectively. Similar trends were observed for isolate C.a 149 of C. acutatum at $10^{4}$, and $10^{5}$, and $10^{6}$ conidia/ml, corresponding to $10.4,8.2$, and 8.0 days, respectively (Table 2 ).

Screening for reduced-pathogenicity mutants. The foliar-dip method was evaluated for detecting reduced pathogenicity of REMI mutants compared with the n-path mutant of C. magna. Significant differences in mortality were observed in seedlings inoculated with the wild-type C.g 342 of C. gloeosporioides compared

Table 1. Time required to reach $50 \%$ mortality in young (12-week-old) and older (15-week-old) strawberry seedlings by the root-soak and foliar-dip methods at different incubation temperatures with isolate C.a 149 of Colletotrichum acutatum and isolate C.g 318 of C. gloeosporioides

\begin{tabular}{lcccc} 
& & & \multicolumn{2}{c}{ Days to 50\% mortality $^{\mathbf{z}}$} \\
\cline { 4 - 5 } Isolate & Inoculation method & Incubation temperature $\left({ }^{\circ} \mathbf{C}\right)$ & Young & Older \\
\hline C.a 149 & Foliar dip & 25 & $8.2 \mathrm{a}$ & $9.2 \mathrm{ab}$ \\
C.a 149 & Root soak & 25 & $7.7 \mathrm{a}$ & $15.3 \mathrm{c}$ \\
C.a 149 & Foliar dip & 19 & $11.2 \mathrm{~b}$ & $8.2 \mathrm{a}$ \\
C.a 149 & Root soak & 19 & $8.2 \mathrm{a}$ & $10.2 \mathrm{~b}$ \\
C.g 318 & Foliar dip & 25 & $9.5 \mathrm{ab}$ & $8.3 \mathrm{ab}$ \\
C.g 318 & Root soak & 25 & $10.5 \mathrm{~b}$ & $14.5 \mathrm{c}$ \\
C.g 318 & Foliar dip & 19 & $9.8 \mathrm{~b}$ & $10.1 \mathrm{~b}$ \\
C.g 318 & Root soak & 19 & $16.9 \mathrm{c}$ & $11.4 \mathrm{~b}$ \\
\hline
\end{tabular}

$\mathrm{z}$ Values for $50 \%$ seedling mortality were calculated from the respective regression equation of disease progress for each seedling age $\times$ inoculation method $\times$ isolate $\times$ incubation temperature combination. Values with the same letters do not differ significantly $(P<0.05)$ according to contrast $t$ test.

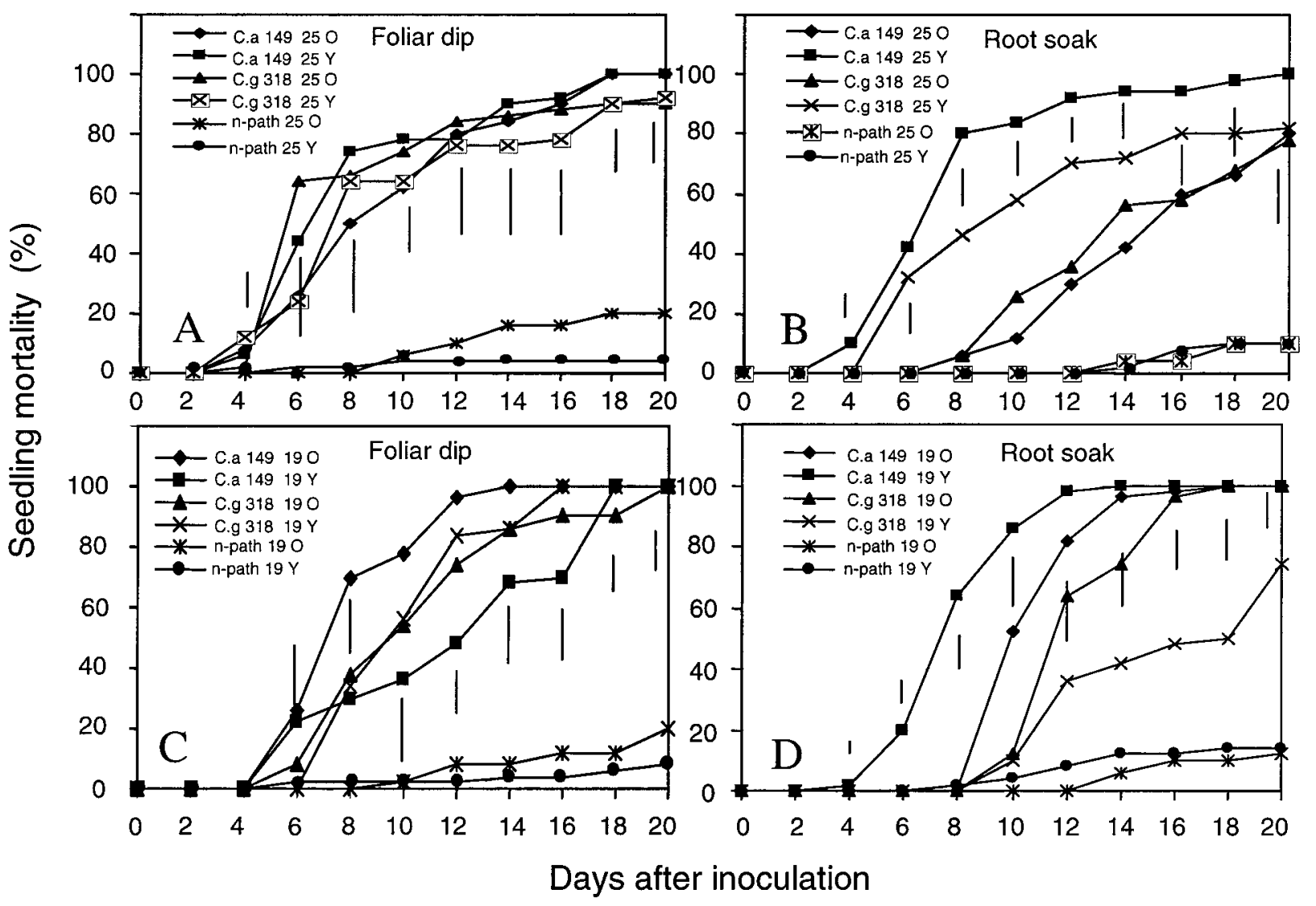

Fig. 2. Disease incidence in strawberry seedlings inoculated with wild-type isolates C.g 318 of Colletotrichum gloeosporioides, C.a 149 of C. acutatum, and nonpathogenic ultraviolet mutant (n-path) of C. magna. A, Foliar dip of young (Y) and older (O) seedlings at $25^{\circ} \mathrm{C}$. B, Root-soak inoculation of $\mathrm{Y}$ and $\mathrm{O}$ seedlings at $25^{\circ} \mathrm{C}$. C, Foliar dip of $\mathrm{Y}$ and $\mathrm{O}$ seedlings at $19^{\circ} \mathrm{C}$. D, Root-soak inoculation of $\mathrm{Y}$ and $\mathrm{O}$ seedlings at $19^{\circ} \mathrm{C}$. Each datum point is the mean of two individual experiments. Distances larger than the vertical bars between symbols at each point are significant $(P \leq 0.05)$ according to Tukey Kramer multiple comparison test. 
with REMI mutant isolates c-52, c-63, e149, and g-233 (Figs. 1B and 3A). Furthermore, wild-type isolate L2.5 of $C$. magna, pathogenic on cucurbits, did not cause disease in strawberry seedlings (Fig. 3A). REMI mutants that exhibited reduced pathogenicity after three seedling screening tests were used to inoculate mature daughter plants. Disease severity values were significantly lower for plants inoculated with c-52, c-63, e149, and g233 compared with wild-type isolate C.g 342 of $C$. gloeosporioides (Table 3).

Assays for germination and appressorium formation. Frequency of appressorium formation was determined and compared with that of the wild-type isolates. Significant differences were observed between the n-path mutant compared with that of wild-type L2.5 isolate of C. magna. Significant differences also were observed between the REMI reducedpathogenicity mutants and the corresponding wild-type C.g 342 isolate of C. gloeosporioides. In n-path and REMI mutants c63, e-149, and g-233, conidia tended to form long germ tubes without appressoria at significantly higher rates than their corresponding wild-type isolates (Fig. 3B). The frequency of conidial germination and initial appressorium formation was significantly higher in wild-type C.g 342 than in reduced-pathogenicity REMI mutants.

\section{DISCUSSION}

As a first step in evaluating pathogenicity of Colletotrichum REMI mutants on strawberry seedlings, it was necessary to develop a rapid, reliable screening protocol. To assess pathogenicity of isolates, it was necessary to clearly define the role of seedling age in susceptibility to anthracnose. Young and older seedlings inoculated by foliar dip were similar in the time required to reach $50 \%$ mortality at $25^{\circ} \mathrm{C}$ (Table 1). Young immature, true leaves are preferentially susceptible to infection by Colletotrichum spp. $(7,21)$. Thus, seedling transplanting, which is time and labor

Table 2. Time required to reach $50 \%$ mortality in young strawberry seedlings inoculated by the foliar-dip method with three different inoculum concentrations of isolates C.a 149 of Colletotrichum acutatum and C.g 318 of C. gloeosporioides

\begin{tabular}{lcc}
\hline Isolate & $\begin{array}{c}\text { Inoculum } \\
\text { (conidia/ml) }\end{array}$ & $\begin{array}{c}\text { Time } \\
\text { (days) }^{\mathbf{z}}\end{array}$ \\
\hline C.g 318 & $10^{4}$ & $13.8 \mathrm{a}$ \\
C.g 318 & $10^{5}$ & $9.5 \mathrm{~b}$ \\
C.g 318 & $10^{6}$ & $6.4 \mathrm{c}$ \\
C.a 149 & $10^{4}$ & $10.4 \mathrm{~d}$ \\
C.a 149 & $10^{5}$ & $8.2 \mathrm{~b}$ \\
C.a 149 & $10^{6}$ & $8.0 \mathrm{~b}$ \\
\hline
\end{tabular}

${ }^{\mathrm{z}}$ Values for $50 \%$ seedling mortality were calculated from the regression equation of disease progress for each inoculum concentration and isolate combination. Values with different letters are significantly different based on results of contrast $t$ tests $(P<0.05)$. consuming, is dispensable for screening pathogenicity by the foliar-dip method. Recent studies have shown plant age to be an important consideration when screening strawberry seedlings for resistance. In general, seedlings become more resistant as they mature (27). In this study, only older seedlings inoculated by the root-soak method seemed to be more resistant at $25^{\circ} \mathrm{C}$, compared with young seedlings (Fig. 1B). This may be the result of a more developed root system in older seedlings. Traditionally, older seedlings are used to screen for cultivar resistance $(7,27,28)$ while screening done at an early age is more rigorous, and may eliminate some mutants for reduced pathogenicity. On the other hand, time, labor, space, and material are the main advantages of the proposed method.

Both the root-soak and foliar-dip methods proved to be useful in pathogenicity assessment. However, inoculation of young and older seedlings by the foliar-dip method allowed us to distinguish increased virulence based on lesion size on leaves and petioles. Although only seedling mortality was monitored using this technique, it was important to assess mutant pathogenicity primarily during the second and third screening stages. Our results are in agreement with those obtained previously by Denoyes-Rothan and Guerin (7) who found that, in order to screen for cultivar resistance to $C$. acutatum, a dip inoculation technique in which entire plants were immersed in a conidial suspension resulted in accurate disease response of susceptible cultivars.

Disease progress was delayed at $19^{\circ} \mathrm{C}$ compared with $25^{\circ} \mathrm{C}$ with all pathogenic isolates (Fig. 2), which was in agreement with previous studies showing that a latent infection period decreased as temperatures increased (17,32). Although appearance of disease symptoms was delayed in seedlings inoculated by the leaf method at $19^{\circ} \mathrm{C}$, disease incidence progressed and eventually reached levels similar to that at $25^{\circ} \mathrm{C}$ (Fig. 2C). An incubation temperature of $19^{\circ} \mathrm{C}$ allowed better discrimination between C.g 318 of C. gloeosporioides and
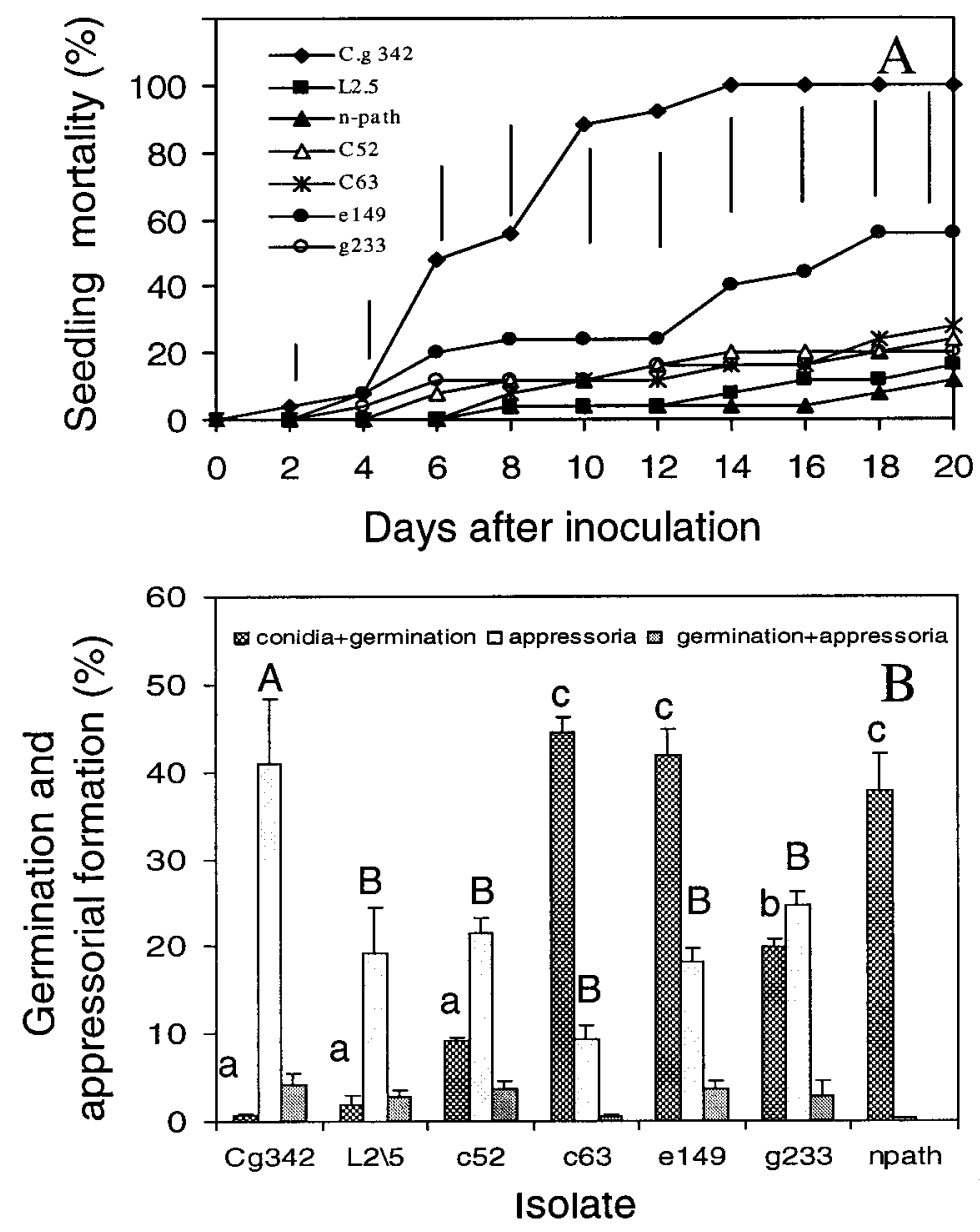

Fig. 3. A, Disease response of strawberry seedlings inoculated with mutant isolates of Colletotrichum gloeosporioides and control isolates L2.5, a nonpathogenic mutant (n-path) of C. magna, and C.g 342 of $C$. gloeosporioides. Distances between symbols at each point that are larger than the vertical bars are significant $(P \leq 0.05)$. B, Assessment of conidial germination and appressorial formation compared to wild-type isolates. Each column in B is the mean of three individual experiments. Bars represent standard error of the mean and common letters are not significantly different $(P \leq 0.05)$, according to the Tukey-Kramer multiple comparison test, for each of the parameters (conidia + germination, appressoria) between isolates. 
C.a 149 of C. acutatum in seedlings inoculated by the root-soak method (Fig. 2D). Recent studies showed that, at lower temperatures, $C$. acutatum produced more conidia than C. gloeosporioides $(17,23)$. However, at $25^{\circ} \mathrm{C}$, there was little difference in disease incidence among control isolates; therefore, $25^{\circ} \mathrm{C}$ was chosen as the recommended incubation temperature.

Conidial concentration is an important factor in inducing the disease response in strawberry seedlings (Table 2). Significant differences in seedling mortality due to different concentrations of C.g 318 may be explained by lower sporulation of $C$. gloeosporioides compared with $C$. acutatum and a shorter latent period of $C$. acutatum compared with $C$. gloeosporioides (17). Initial inoculum concentration may have changed over time during incubation and may be important in such a short pathogenicity bioassay. A conidial suspension adjusted to $10^{5} \mathrm{conidia} / \mathrm{ml}$ was effective in screening for reduced pathogenicity mutants, and well suited for the purpose of these screening tests.

The root-soak and the foliar-dip methods allowed for discrimination of nonspecific host-pathogen interaction, whereby the specific cucurbit pathogen, C. magna, did not cause disease in strawberry seedlings at the conidial concentrations tested (Fig. 3A). In order to screen for reduced pathogenicity mutants, we chose the technique causing typical symptoms on susceptible cultivars that required less time and labor (i.e., foliage inoculation of young seedlings using inoculum concentration adjusted to $10^{5}$ conidia/ml at an incubation temperature of $25^{\circ} \mathrm{C}$ ). This method was consistent for evaluation of pathogenicity of Colletotrichum REMI mutants on strawberry seedlings. The developed technique was reliable and accurate because isolates that were reduced or impaired in pathogenicity on young seedlings during several screenings were re-

Table 3. Response of mature strawberry daughter plants to inoculation with various restriction enzyme-mediated integration mutants of Colletotrichum gloeosporioides and C. acutatum reduced in pathogenicity relative to wild-type isolates

\begin{tabular}{ll}
\hline Isolates & DSR $^{\mathbf{z}}$ \\
\hline C.g 342 & $4.25 \mathrm{a}$ \\
N-path & $0.00 \mathrm{~b}$ \\
c-52 & $0.50 \mathrm{~b}$ \\
c-63 & $1.25 \mathrm{~b}$ \\
e-149 & $0.75 \mathrm{~b}$ \\
g-233 & $0.25 \mathrm{~b}$ \\
\hline
\end{tabular}

${ }^{\mathrm{z}}$ Disease severity rating (DSR) of daughter plants was scored 2 weeks after inoculation for disease symptoms according to a 0-to-5 scale, where $0=$ no visible lesions and $5=$ dead plants; values represent the means for 10 plants per isolate. Values with different letters are significantly different at $P \leq 0.05$ according to the Tukey-Kramer multiple comparison test. duced consistently or nonpathogenic on plants at all developmental stages, including mature daughter plants (Fig 3A; Table 3 ). Therefore, this method is recommended for a preliminary screening of a large population of transformants. The proposed method requires considerably less space because Eppendorf tubes are used instead of pots. The rapid screening of potential mutants could not have been performed with such ease with the standard spray inoculation techniques $(7,27,28)$. The foliar-dip method was devised for largescale screening and isolation of Colletotrichum REMI mutants with reduced pathogenicity. The time required for screening 150 mutants with this procedure, which involves two people, was approximately $8 \mathrm{~h}$.

Five of the REMI mutants impaired in pathogenicity isolated by the rapid technique were characterized for initial parameters of germination and appressorial formation. Formation of the appressorium and penetration of the host are integral parts of the infection process of Colletotrichum spp. (24). In some species, formation of appressoria may be obligatory for infection; whereas, in others, it may be optional or not required at all (35). A mode of germination observed for REMI mutants c-63, e-149, and g-233 consisting of extended germ tube elongation before appressorium formation or the absence of appressorium formation also has been reported for Cochliobolus heterostrophus (5) and Magnaporthe grisea (30). Colletotrichum acutatum shows similar germination behavior on asymptomatic plants (eggplant, pepper, and tomato) which occurred mainly from one side of the conidium and was characterized by long, thin, unbranched hyphae (14). This kind of development occasionally resembles the effect of mutation in signal transduction components observed in other fungal species $(1,34)$. Further characterization of the reduced-pathogenicity mutants will allow us to identify specific genes involved in pathogenicity of Colletotrichum in strawberry.

In this study, a large-scale screening technique was developed for pathogenicity assessment of Colletotrichum isolates and its benefit for selection of reduced-virulence mutants was proven. Application of this procedure may have potential for screening additional pathogens on strawberry, such as Rhizoctonia spp., and for assessing potential biocontrol agents.

\section{ACKNOWLEDGMENTS}

We thank Y. Nitzani and M. Maymon for technical assistance.

\section{LITERATURE CITED}

1. Adachi, K., and Hamer, J. E. 1998. Divergent cAMP signaling pathways regulate growth and pathogenesis in the rice blast fungus Magnaporthe grisea. Plant Cell 10:1361-1373.

2. Akamatsu, H., Itoh, Y., Kodama, M., Otani, H., and Kohmoto, K. 1997. AAL toxin deficient mutants of Alternaria alternata tomato pathotype by restriction enzyme mediated integration. Phytopathology 87:967-972.

3. Bolker, M., Bohnert, H. U., Braun, K. H Gorl, J., and Kahmann, R. 1995. Tagging pathogenicity genes in Ustilago maydis by restriction enzyme mediated integration (REMI) Mol. Gen. Genet. 248:547-552.

4. Curry, K. J., Abril, M., Avant, J. B., and Smith, B. J. 2002. Strawberry anthracnose: Histopathology of Colletotrichum acutatum and C. fragariae. Phytopathology 92:1055-1063.

5. Dean, R. A. 1997. Signal pathways and appressorium morphogenesis. Annu. Rev. Phytopathol. 35:211-234.

6. Denoyes, B., and Baudry, A. 1995. Species identification and pathogenicity study of French Colletotrichum strains isolated from strawberry using morphological and cultural characteristics. Phytopathology 85:53-57.

7. Denoyes-Rothan, B., and Guerin, M. 1996 Comparison of six inoculation techniques with Colletotrichum acutatum on cold stored strawberry plants and screening for resistance to this fungus in French strawberry collection. Plant Pathol. 102: 615-621.

8. Denoyes-Rothan, B., Lafargue, M. Guerin, G., and Clerjeau, M. 1999. Fruit resistance to Colletotrichum acutatum in strawberries. Plant Dis. 83:549-553.

9. Eastburn, D. M., and Gubler, W. D. 1990. Strawberry anthracnose: detection and survival of Colletotrichum acutatum in soil. Plant Dis. 74:161-163.

10. Freeman S., Horowitz, S., and Sharon A. 2001. Pathogenic and nonpathogenic lifestyles in Colletotrichum acutatum from strawberry and other plants. Phytopathology 91:986-992.

11. Freeman, S., and Katan, T. 1997. Identification of Colletotrichum species responsible for anthracnose and root necrosis of strawberry in Israel. Phytopathology 87:516-521.

12. Freeman, S., and Rodriguez, R. J. 1992. A rapid reliable, bioassay for pathogenicity of Colletotrichum magna on cucurbits and its use in screening for nonpathogenic mutants. Plant Dis. 76:901-905.

13. Freeman, S., Shalev, Z., and Katan J. 2002. Survival in soil of Colletotrichum acutatum and $C$. gloeosporioides pathogenic on strawberry. Plant Dis. 86:965-970.

14. Horowitz, S., Freeman, S., and Sharon, A. 2002. Use of green fluorescent protein transgenic strains to study pathogenic and nonpathogenic lifestyles in Colletotrichum acutatum. Phytopathology 92:743-749.

15. Howard, C. M., and Albregts, E. E. 1983. Black leaf spot phase of strawberry anthracnose caused by Colletotrichum gloeosporioides (C. fragariae). Plant Dis. 67:11441146.

16. Howard, C. M., Maas, J. L., Chandler, C. K. and Albregts, E. E. 1992. Anthracnose of strawberry caused by the Colletotrichum complex in Florida. Plant Dis. 76:976-981.

17. King, W. T., Madden, L. V., Ellis, M. A., and Wilson, L. L. 1997. Effects of temperature on sporulation and latent period of Colletotrichum spp. infecting strawberry fruit. Plant Dis. 81:77-84.

18. Koch-Dean, M., Taanami, Z., and Freeman, S. 2002. Development of strawberry cultivars resistant to anthracnose (Colletotrichum acutatum) in Israel. In: Proc. Fourth Int. Strawberry Symp. T. Hietaranata, M. M. Linna, P. Palonen, and P. Parikka, eds. Acta Hortic. 567:97-99.

19. Kuspa, A., and Loomis, W. F. 1992. Tagging developmental genes in Dictyostelium by restriction enzyme mediated integration of plasmid DNA. Proc. Natl. Acad. Sci. USA 89:8803-8807.

20. Leandro, L. F. S., Gleason, M. L., Nutter, F. W., Jr., Wegulo, S. N., and Dixon, P. M. 2001. 
Germination and sporulation of Colletotrichum acutatum on symptomless strawberry leaves. Phytopathology 91:659-664.

21. Legard, D. E. 2000. Colletotrichum diseases of strawberries in Florida. Pages 292-299 in: Colletotrichum: Host Specificity, Pathology, and Host Pathogen Interaction. D. Prusky, S. Freeman, and M. B. Dickman, eds. American Phytopathological Society Press, St. Paul, MN.

22. Maas, J. L., and Howard, C. M. 1985. Variation of several anthracnose fungi in virulence to strawberry and apple. Plant Dis. 69:164166.

23. Ntahimpera, N., Wilson, L. L., Ellis, M. A., and Madden, L. V. 1999. Comparison of rain effect on splash dispersal of three Colletotrichum species infecting strawberry. Phytopathology 89:555-563.

24. O'Connell, R., Perfect, S., Hughes, B., Carazaniga, R., Bailey, J., and Green, J. 2000. Dissecting the cell biology of Colletotrichum infection processes. Pages 57-77 in: Colletotrichum: Host Specificity, Pathology and Host Pathogen Interaction. D. Prusky, S. Freeman, and M. B. Dickman, eds. American Phytopathological Society Press, St. Paul, MN.
25. Robinson, M., and Sharon, A. 1999. Transformation of the bioherbicide Colletotrichum gloeosporioides f. sp. aeschynomene by electroporation of germinated conidia. Curr. Genet. 36:98-104.

26. Smith, B. J., and Black, L. L. 1990. Morphological, cultural, and pathogenic variation among Colletotrichum species isolated from strawberry. Plant Dis. 74:69-76.

27. Smith B. J., Black, L. L., and Galletta, G. J. 1990. Resistance to Colletotrichum fragariae in strawberry affected by seedling age and inoculation method. Plant Dis. 74:1016-1021.

28. Smith, B. J., and Spiers, J. M. 1982. Evaluating techniques for screening strawberry seedlings for resistance to Colletotrichum fragariae. Plant Dis. 66:559-561.

29. Sweigard, J. A., Carroll, A. M., Farrall, L., Chumley, F. G., and Valent, B. 1998. Magnaporthe grisea pathogenicity genes obtained through insertional mutagenesis. Mol. PlantMicrobe Interact. 11:404-412.

30. Talbot, N. J., Ebbole, D. J., and Hamer, J. E. 1993. Identification and characterization of MPG1, a gene involved in pathogenicity from the rice blast fungus Magnaporthe grisea.
Plant Cell 5:1575-1590.

31. Thon, M. R., Nuckles, E. M., and Vaillancourt, L. J. 2000. Restriction enzyme-mediated integration used to produce pathogenicity mutants of Colletotrichum graminicola. Mol. PlantMicrobe Interact. 13:1356-1365.

32. Wilson, L. L., Madden, L. V., and Ellis, M. A. 1990. Influence of temperature and wetness duration on infection of immature and mature strawberry fruit by Colletotrichum acutatum. Phytopathology 80:111-116.

33. Yakoby, N., Zhou, R., Kobiler, I., Dinoor, A and Prusky, D. 2001. Development of Colletotrichum gloeosporioides restriction enzymemediated integration mutants as biocontrol agents against anthracnose disease in avocado fruits. Phytopathology 91:143-148.

34. Yang, Z., and Dickman, M. B. 1999. Colletotrichum trifolii mutants disrupted in the catalytic subunit of cAMP dependent protein kinase are nonpathogenic. Mol. Plant-Microbe Interact. 12:430-439.

35. Zulfigar, M., Brlansky, R. H., and Timmer, L. W. 1996. Infection of flower and vegetative tissues of citrus by Colletotrichum acutatum and C. gloeosporioides. Mycologia 88:121-128. 